\title{
Balanceo de Ecuaciones Químicas Integrando las Asignaturas de Química General, Algebra Lineal y Computación: Un Enfoque de Aprendizaje Activo
}

\author{
Alejandro Regalado-Méndez, Fátima K. Delgado-Vidal, Roberto E. Martínez-López y Ever Peralta-Reyes \\ Universidad del Mar, Campus Puerto Ángel, Ciudad Universitaria S/N, 70902, Puerto Ángel, San Pedro \\ Pochutla, Oaxaca, México. (e-mail: alejandro.regalado33@gmail.com; fatima@angel.umar.mx; \\ martinez@angel.umar.mx; pere@ angel.umar.mx)
}

Recibido Nov. 8, 2013; Aceptado Dic. 31, 2013; Versión final recibida Feb. 27, 2014

\begin{abstract}
Resumen
Se diseñó una estrategia de aprendizaje activo que integra la curricula del plan de estudios de Ingeniería en Acuicultura de la Universidad del Mar en México. Dicha estrategia consiste en que los estudiantes desarrollen un programa en MATLAB ${ }^{\circledR}$ que les permita balancear ecuaciones químicas mediante el método algebraico. Los resultados más importantes de esta investigación son que el grado de aprendizaje de los alumnos respecto a los conceptos básicos en el balanceo de ecuaciones químicas fue de medio a alto; y que el promedio de desarrollo de las capacidades que requerirán los alumnos en el mundo laboral fue de bajo a medio. También se identificó que los alumnos aumentaron su creatividad respecto a la solución de problemas complejos. El porcentaje de alumnos acreditados para el ciclo 2013 es del $100 \%$ y $83.33 \%$ en las asignaturas de computación y química general respectivamente.
\end{abstract}

Palabras clave: aprendizaje activo, balanceo de ecuaciones químicas, enseñanza aprendizaje, método químico, método algebraico

\section{Balancing of Chemical Equations by Integrating General Chemistry, Linear Algebra and Computer Programming: An Active Learning Approach}

\begin{abstract}
An active learning strategy that integrates the curriculum of aquaculture engineering at Del Mar University in Mexico was designed. Such strategy implies that the students develop MATLAB ${ }^{\circledR}$ codes to compute stoichiometric coefficients and balance chemical equations by algebraic methods. The most significant results of this research are that the degree of learning about the fundamentals of balancing chemical equations was from average to high; and average development of the capacities that the student will need in their future work, was from low to average. It was also identified that students increased their creativity regarding troubleshooting. The percentage of students certified for cycle 2013 is $100 \%$ and $83.33 \%$ in the subjects of computing and general chemistry respectively.
\end{abstract}

Keywords: active learning, balancing chemical equations, teaching-learning, chemical method, algebraic method 


\section{INTRODUCCIÓN}

La enseñanza de ciencias e ingenierías se basa frecuentemente en conferencias como método de instrucción (Ramaswamy et al., 2001). Este método tradicional no estimula a los estudiantes a tener una participación activa en su aprendizaje, profundidad en el entendimiento de los conceptos fundamentales, en la aplicación de dichos conceptos para la resolución activa de problemas y mucho menos en el diseño de nuevos productos o diseños. Lo antes mencionado se debe a muchas razones, en las cuales se incluyen la mala atención de los alumnos, ejemplos muy simplificados y sencillos, y demasiado material presentado en un corto plazo (González et al., 2012). Felder y Brent (2003) comentan que los métodos tradicionales centrados en el profesor han demostrado menor eficacia que la enseñanza centrada en el estudiante o en el aprendizaje activo. Por otro lado Prince (2004), Middleton (2013) y Blasco-Arcas et al. (2013) comentan que una verdadera participación activa del estudiante en el proceso de aprendizaje se logra a través de la resolución de problemas, trabajo en equipo, debates, autoevaluación y evaluación por pares, y todas aquellas actividades que requieran los estudiantes para aplicar lo que están aprendiendo, tanto dentro como fuera de la clase.

El Aprendizaje Activo puede ser una buena alternativa para generar un cambio del pensamiento tradicional de los ingenieros a un pensamiento holístico. El Aprendizaje Activo data desde los tiempos de Sócrates, quien promovió el pensamiento crítico y reflexivo a través de la estimulación de interrogatorios. Esta técnica consiste en que los estudiantes incrementen su capacidad de retención de la información tras el período de clase (Keyser, 2000); es ampliamente aceptada hoy en día como una forma de calidad de la educación, el cual se ha implementado en los diferentes niveles educativos (por ejemplo: la escuela primaria, escuela secundaria, escuela politécnica, universidades, organismos de comercio profesionales, y la educación continua) y en diferentes instituciones de educación superior de corte internacional, entre ellas incluyen China, Inglaterra, Australia, Estados Unidos, España y México. Varios investigadores incluyendo a Blottnitz (2006), Bullard y Felder (2007), Evans et al. (2008), El-Naas (2011), Arnaldos et al. (2012), han demostrado que el Aprendizaje Activo es excelente método de enseñanza en programas de ingeniería (por ejemplo: Ingeniería eléctrica, Ingeniería química, entre otros) ya que han logrado una participación activa de los estudiantes en su proceso de enseñanza, memoria de largo plazo respecto a conceptos fundamentales, así como el fortalecimiento de sus habilidades genéricas (tales como; trabajo en grupo, presentación oral, pensamiento crítico y reflexivo).

Prince (2004) y Felder y Brent (2009) proporcionan definiciones de Aprendizaje Activo. Sin embargo los autores tomamos aquélla proporcionada por Açikgöz (2006) en la cual se enuncia que: "Un Aprendizaje Activo es aquel proceso donde el alumno toma la responsabilidad del proceso de aprendizaje, viéndose obligado a usar su capacidad intelectual, ofreciéndole la oportunidad de tomar decisiones sobre los diversos aspectos del proceso de aprendizaje y se le permite hacer la autorregulación". Con este tipo de aprendizaje los estudiantes pueden formar grupos con roles definidos para cada estudiante y una tarea que el grupo pueda llevar a cabo (Tedesco-Schneck, 2013).

Prince (2004) comenta que el Aprendizaje Activo puede ser dividido en Aprendizaje Colaborativo, Aprendizaje Cooperativo y el Aprendizaje Basado en Problemas (ABP). En primera instancia, el Aprendizaje Colaborativo es "referido a cualquier método de enseñanza en el que los estudiantes trabajan juntos en pequeños grupos hacia un objetivo común". Mientras que el Aprendizaje Cooperativo se puede definir como "una forma estructurada de trabajo en grupo donde los estudiantes persiguen objetivos comunes, marcando como diferencia la evaluación de forma individual". Finalmente, el ABP es "un método de instrucción donde se introducen problemas relevantes al inicio del ciclo de instrucción y utilizan un contexto matizado para brindar la motivación del aprendizaje". Este último método de enseñanza siempre es activo y por lo general colaborativo (pueden existir excepciones).

La Secretaría de Educación Pública (SEP) exige a la Universidad del Mar (UMAR) brindar educación de alta calidad a los estudiantes. Por lo que solicita la acreditación de la oferta educativa ante el Comité Interinstitucional para la Evaluación de la Educación Superior (CIEES) y/o el Consejo de Acreditación de la Enseñanza de la Ingeniería (CACEI). Esto lleva al objetivo de aumentar la matricula estudiantil de la UMAR para obtener más recursos económicos del gobierno federal.

En la experiencia docente de los autores impartiendo los cursos de química general y computación en ocho ciclos (2005 a 2012) consecutivos en la carrera de Ingeniería en Acuicultura de la UMAR, se ha encontrado que el porcentaje de alumnos no acreditados en el curso de química general oscila entre el $38 \%$ y el $75 \%$, mientras que en la asignatura de computación el porcentaje oscila entre el $25 \%$ a $50 \%$. Estas cifras son preocupantes considerando que los grupos son reducidos (dos a once alumnos). Por otro lado, los alumnos de la asignatura de química general muestran una negación extrema de aprender esta ciencia por considerar el curso difícil de comprender en sus conceptos básicos y el no poder relacionarlo con su campo 
de acción. Con este antecedente la enseñanza de la química y computación dentro del programa de Ingeniería en Acuicultura ofertado en la UMAR representa un gran reto para los autores.

Finalmente, los autores queremos mencionar que no solo tomamos en cuenta las exigencias de la SEP y autoridades de la UMAR, sino que también estamos de acuerdo con la declaración de Bologna (1999), en la cual se muestra la necesidad de reorganizar la curricula en la educación superior para brindar una educación de alto nivel.

Por todo lo anterior, este trabajo pretende contribuir al fortalecimiento de la Carrera de Ingeniería en Acuicultura, brindando educación de alto nivel a sus estudiantes, así mismo reducir el índice de reprobación en las asignaturas de química general y computación del programa de estudios de dicha carrera, así como promover el desarrollo de las competencias demandadas en el mundo laboral (tabla 1). Lo anterior es completado mediante el diseño y aplicación de una estrategia de enseñanza-aprendizaje basada en el constructivismo, específicamente en el Aprendizaje Activo. Dicha estrategia consta de elementos del Aprendizaje Cooperativo, Aprendizaje Colaborativo y elementos del Aprendizaje Basado guiado.

Tabla 1: Competencias genéricas y profesionales deseadas en estudiantes de ingeniería con base González y González (2008). Números arábigos 1-7: Numero de competencia; Aprendizaje: A; Personales: P; Autonomía: AU y Valores: V.

\begin{tabular}{|c|c|c|c|}
\hline Aprendizaje & Personales & Autonomía & Valores \\
\hline $\begin{array}{l}\text { 1. Abstracción, análisis y } \\
\text { síntesis } \\
\text { 2. Investigación científica } \\
\text { 3. Aplicación práctica de } \\
\text { conocimientos básicos } \\
\text { 4. Organización y } \\
\text { planificación del tiempo } \\
\text { 5. Aprendizaje autónomo y } \\
\text { actualización } \\
\text { 6. Buscar, procesar y } \\
\text { analizar información } \\
\text { 7. Identificar, plantear y } \\
\text { resolver problemas }\end{array}$ & $\begin{array}{l}\text { 1. Comunicación oral y } \\
\text { escrita } \\
\text { 2. Trabajo en equipo } \\
\text { 3. Motivación, conducción a } \\
\text { metas comunes } \\
\text { 4. Iniciativa } \\
\text { 5. Asertividad }\end{array}$ & $\begin{array}{l}\text { 1. Pensamiento crítico y } \\
\text { lógico } \\
\text { 2. Creatividad } \\
\text { 3. Solución de problemas } \\
\text { 4. Toma de decisiones } \\
\text { 5. Trabajo individual } \\
\text { 6. Formulación y gestión de } \\
\text { proyectos }\end{array}$ & $\begin{array}{l}\text { 1. Responsabilidad y } \\
\text { honestidad } \\
\text { 2. Compromiso ético } \\
\text { 3. Compromiso a la calidad } \\
\text { 4. Tolerancia y respeto a la } \\
\text { diferencia de opinión } \\
\text { 5. Preservación del } \\
\text { medioambiente }\end{array}$ \\
\hline
\end{tabular}

\section{METODOLOGÍA}

Primeramente se describe el problema que los alumnos van a resolver. El problema presentado tiene la forma tradicional. Sin embargo, para darle respuesta a dicho problema, el instructor conducirá la solución con base en la estrategia de enseñanza-aprendizaje propuesta más adelante (ver Parte I, II y III).

\section{Descripción del Problema}

Se elige el método algebraico como tema central para la enseñanza-aprendizaje de los alumnos dado que permite integrar muy bien la química con la computación. El problema para los alumnos es el siguiente: Dadas las ecuaciones químicas de la Tabla 2, balancear cada una de las ecuaciones químicas por el método algebraico desarrollando un programa en MATLAB ${ }^{\circledR}$. Comentar acerca de sus limitaciones y comparar el método algebraico con los métodos químicos.

Tabla 2: Reacciones Químicas a Balancear.

\begin{tabular}{|c|l|}
\hline 1 & $\mathrm{HNO}_{2}+\mathrm{KMnO}_{4}+\mathrm{H}_{2} \mathrm{SO}_{4} \rightarrow \mathrm{HNO}_{3}+\mathrm{MnSO}_{4}+\mathrm{K}_{2} \mathrm{SO}_{4}+\mathrm{H}_{2} \mathrm{O}$ \\
\hline 2 & $\mathrm{LiCl}+\mathrm{KMnO}_{4}+\mathrm{H}_{2} \mathrm{SO}_{4} \rightarrow \mathrm{Cl}_{2}+\mathrm{MnSO}_{4}+\mathrm{K}_{2} \mathrm{SO}_{4}+\mathrm{Li}_{2} \mathrm{SO}_{4}+\mathrm{H}_{2} \mathrm{O}$ \\
\hline 3 & $\mathrm{~K}_{2} \mathrm{Cr}_{2} \mathrm{O}_{7}+\mathrm{FeCl}_{2}+\mathrm{HCl} \rightarrow \mathrm{KCl}+\mathrm{CrCl}_{3}+\mathrm{FeCl}_{3}+\mathrm{H}_{2} \mathrm{O}$ \\
\hline 4 & $\mathrm{SrCl}_{2}+\mathrm{KMnO}_{4}+\mathrm{H}_{3} \mathrm{PO}_{4} \rightarrow \mathrm{Cl}_{2}+\mathrm{Mn}_{3}\left(\mathrm{PO}_{4}\right)_{2}+\mathrm{Sr}_{3}\left(\mathrm{PO}_{4}\right)_{2}+\mathrm{K}_{3} \mathrm{PO}_{4}+\mathrm{H}_{2} \mathrm{O}$ \\
\hline
\end{tabular}




\section{Diseño del Aprendizaje Activo}

El diseño de la estrategia de enseñanza-aprendizaje consta de 3 sesiones (Parte I, II y III) de trabajo con tiempos de 2 a 3 horas cada una. Estas sesiones deben desarrollarse con la mínima participación del profesor, actuando éste como un facilitador del conocimiento. Dicha estrategia está basada en el método socrático (preguntas-respuestas). Después de cada sesión los alumnos deben escribir un reporte contestando a cada una de las preguntas realizadas. El reporte tiene la finalidad de promover y estimular en los estudiantes las competencias A2, A3, A6-A7, P1-P4, AU1-AU3, y V1-V3.

\section{Parte I}

Esta sección es conducida mediante el aprendizaje colaborativo, de tal forma que se promuevan las competencias A1-A7, P2-P5, AU1-AU5 y V1. Así mismo el facilitador debe de motivar a los estudiantes a investigar lo relacionado con las reacciones químicas. En este sentido, los autores proponemos las siguientes preguntas: ¿Qué es una reacción química?, ¿qué es una ecuación estequiométrica?, ¿cuáles son los tipos de reacciones químicas?, ¿dónde las encontramos?, ¿cómo se balancean las reacciones químicas? y ¿por qué debemos balancear las reacciones químicas?

Una vez que el facilitador promueve el trabajo colaborativo, los estudiantes primeramente tienen 20 minutos para comentar entre ellos acerca de los cuestionamientos realizados, pudiendo emplear la técnica de lluvia de ideas, mapas conceptuales, etc. En segundo término, los estudiantes tienen 30 minutos para realizar una búsqueda de la información en la biblioteca o internet aprovechando la tecnología e instalaciones Wi-Fi para posteriormente discriminar la información entre páginas web, enciclopedias, ebooks, artículos, manuales de universidades reconocidas, etc. Posteriormente, los alumnos cuentan con 30 minutos para escribir un reporte parcial con sus respuestas y/o definiciones propias. Finalmente, en un lapso de 20-30 minutos comparan sus respuestas entre grupos, brindándose comentarios entre ellos.

\section{Parte II}

Esta sección es conducida mediante el aprendizaje guiado, en donde el facilitador inducirá la parte matemática a los estudiantes y promoverá las competencias A1-A7, P2-P4, AU1-AU3, AU5 y V1. La matemática principal consta de la teoría de sistemas de ecuaciones lineales. Para ello, los autores proponemos las siguientes preguntas: ¿qué es un sistema de ecuaciones lineales?, ¿cómo se representa un sistema de ecuaciones lineales?, ¿cómo se clasifican los sistemas de ecuaciones lineales?, ¿cómo se resuelve un sistema de ecuaciones lineales? y ¿dónde encontramos sistemas de ecuaciones lineales?

El facilitador deberá de hacer las preguntas propuestas de forma individual para impulsar a los estudiantes a la escritura de mapas conceptuales, a través de los cuales se debe llegar a una definición de sistemas de ecuaciones, su clasificación y métodos de solución, requiriendo un tiempo aproximado de 40 minutos. Posteriormente, el facilitador debe inducir las propiedades matriciales de los sistemas de ecuaciones lineales, actividad que puede durar 10 minutos. Consecutivamente, el facilitador motiva a realizar los planteamientos de los sistemas de ecuaciones lineales que brinden solución al balanceo de las ecuaciones químicas dadas en el problema, actividad que puede durar hasta 30 minutos. Posteriormente, los alumnos tienen 20 minutos para escribir un reporte parcial con sus respuestas y/o definiciones propias; y finalmente en un lapso de 10-20 minutos comparan sus respuestas entre grupos, brindándose comentarios entre ellos.

Parte III

Esta última sección es conducida mediante el aprendizaje cooperativo para aplicar la teoría de la programación, basándose en diagramas de flujo para diseñar un programa que resuelva los sistemas de ecuaciones lineales generados al implementar el método algebraico en el balanceo de reacciones químicas. Con esta actividad se promueven las competencias A1, A3, A7, P1, P3-P5, AU1-AU5 y V3-V4. Para lograr lo anterior los autores proponemos que el programa pueda realizar: i) Leer la matriz de coeficientes $(\underset{\sim}{A})$ del sistema de ecuaciones lineales y del vector solución $(\underset{\sim}{b})$ directamente con el teclado, $O$ un archivo con extensión dat, txt o cvs; ii) calcular el rango de las matrices $\underset{\sim}{A}$ y $\underset{\sim}{B}$ vía MATLAB® para caracterizar y predecir qué tipo de solución tiene el sistema de ecuaciones lineales; iii) resolver el sistema de ecuaciones lineales y iv) que muestre solo coeficientes enteros y el menor múltiplo deseado.

Para promover el trabajo cooperativo, cada estudiante cuenta con 15 minutos para realizar una búsqueda de manera individual acerca de definiciones de algoritmos en la biblioteca o internet para posteriormente discriminar información entre páginas web, enciclopedias, ebooks, artículos, manuales de universidades reconocidas. Después, los alumnos tienen 20 minutos para desarrollar un algoritmo y/o pseudocódigo que 
les permita dar solución a los sistemas de ecuaciones lineales planteados. Posteriormente, los miembros de cada equipo discuten sus algoritmos diseñados, seleccionando el algoritmo que les parezca correcto. Consecuentemente, en 20 minutos deberán pasar el algoritmo a lenguaje de máquina, específicamente el programa deberá ser escrito en MATLAB ${ }^{\circledR}$, del cual pueden emplear las herramientas incluidas en el software. Finalmente, los alumnos tienen 20 minutos más para escribir un reporte parcial con sus respuestas propias y en un lapso de 10 minutos comparar sus respuestas entre grupos, aportándose comentarios entre ellos.

\section{Evaluación de la Actividad de Aprendizaje Activo}

La actividad de aprendizaje activo es evaluada midiendo los siguientes aspectos: a.- Grado de integración de conocimientos para el balanceo de reacciones químicas, b.- Grado de cumplimiento en la solución del problema planteado, c.- Grado de cumplimiento del formato del reporte entregado y d.- Desarrollo de las capacidades del mundo laboral (aprendizaje, personales, autonomía y valores)

\section{a.- Grado de integración de conocimientos para el balanceo de reacciones químicas}

i) Identificación de una reacción REDOX (oxidación-reducción) a través de los estados de oxidación de los átomos de la reacción a balancear; ii) planteamiento de sistemas de ecuaciones lineales vía la interpretación de la matriz atómica de la reacción a balancear; iii) diseño de un diagrama de flujo y/o pseudocódigo; iv) solución correcta de los sistemas de ecuaciones lineales de la ecuación química a balancear vía código en MATLAB $^{\circledR}$; v) balanceo de ecuaciones REDOX por el método del ión-electrón o por el cambio en el número de oxidación.

b.- Grado de cumplimiento en la solución del problema planteado

i) Balancear cada una de las ecuaciones químicas por el método algebraico desarrollando un programa en MATLAB ${ }^{\circledR}$; ii) comentar acerca de las limitaciones del método algebraico; iii) comparar el método algebraico con los métodos químicos.

c.- Grado de cumplimiento del formato del reporte entregado

i) Estructura del reporte (resumen, marco teórico, metodología, resolución del ejercicio, conclusiones y referencias bibliográficas); ii) calidad del reporte, la cual es evaluada con base en los criterios establecidos por Gavilán et al. (2013); y iii) entrega del reporte en tiempo y forma.

d.- Desarrollo de las capacidades del mundo laboral (aprendizaje, personales, autonomía y valores)

Estas capacidades serán calculadas según la percepción del facilitador en cuanto a la calidad del trabajo por equipo e individual.

La escala de calificación es de 0 a 3, la cual es similar a la escala de Likert, entendiendo como 0: nulo; 1: bajo; 2: medio; y 3: alto.

\section{Participantes}

En la actividad de aprendizaje activo propuesta en esta investigación participaron seis estudiantes (aproximadamente 19 años de edad, dos mujeres y cuatro hombres) de licenciatura del programa de Ingeniería en Acuicultura de la UMAR, quienes tomaban las clases de computación y química en el mismo semestre.

\section{RESULTADOS Y DISCUSIONES}

Los alumnos adquirieron e integraron los conocimientos relacionados con el balanceo de ecuaciones químicas, algebra lineal y computación. De tal forma que los dos equipos lograron identificar que las reacciones eran del tipo REDOX, así como integrar completamente los conocimientos básicos requeridos para desarrollar el método algebraico y por ende el programa MATLAB ${ }^{\circledR}$. Lo anterior fue reflejado en el hecho que las cuatro ecuaciones químicas fueron balanceadas correctamente. En la Tabla 3 se presentan los resultados obtenidos al evaluar el grado de integración de conocimientos al balancear las reacciones químicas. El promedio de la evaluación los equipos A y B fue 2.5 y 3.0 respectivamente, mostrando una desviación estándar de los equipos $A$ y $B$ de 0.547 y 0.0 respectivamente. Por tanto el equipo $A$ integró sus conocimientos en grado alto. 
Tabla 3: Grado de integración de conocimientos para el balanceo de reacciones químicas.

\begin{tabular}{|l|c|c|}
\hline \multirow{2}{*}{ Conocimiento } & \multicolumn{2}{|c|}{ Grado de integración } \\
\cline { 2 - 3 } & \multicolumn{2}{|c|}{ Equipo } \\
\hline $\begin{array}{l}\text { Identificación de una reacción REDOX por determinación del estado de oxidación de los } \\
\text { átomos }\end{array}$ & 2 & 3 \\
\hline $\begin{array}{l}\text { Planteamiento de sistemas de ecuaciones lineales mediante la matriz atómica de la } \\
\text { ecuación química }\end{array}$ & 2 & 3 \\
\hline Diseño diagrama de flujo y/o pseudocódigo & 3 & 3 \\
\hline Diseño del código en MATLAB & 3 & 3 \\
\hline $\begin{array}{l}\text { Balanceo de ecuaciones REDOX por el método de ión-electrón y/o por el cambio en el } \\
\text { número de oxidación }\end{array}$ & 2 & 3 \\
\hline Solución correcta de las ecuación químicas & 3 & 3 \\
\hline Promedio & 2.500 & 3.000 \\
\hline Desviación estándar & 0.547 & 0.000 \\
\hline
\end{tabular}

Ambos equipos balancearon acertadamente las cuatro ecuaciones químicas por el método algebraico con ayuda del programa desarrollado en MATLAB ${ }^{\circledR}$. Sin embargo omitieron en sus reportes las limitaciones de dicho método; a pesar de que argumentaron la buena utilidad de MATLAB ${ }^{\circledR}$, refiriendo que pueden balancear las ecuaciones mediante pocas instrucciones y que ahorra bastante tiempo en el balanceo de las ecuaciones químicas. Con respecto a la comparación del método algebraico con otros métodos químicos, ambos equipos hicieron dicha comparación, aunque el equipo $A$ en menor grado al no resolver la ecuación cuatro por ningún otro método. Por su parte, el equipo B comparó el método algebraico contra el método del ión-electrón, obteniendo los mismos resultados por ambos métodos para las cuatro ecuaciones químicas. En la Tabla 4 se muestra el grado de cumplimiento en la solución del problema planteado. Los promedios en cuanto a este grado de cumplimiento para los quipos A y B fueron 1.667 y 2.0 respectivamente con desviación estándar de 1.527 (equipo A) y 1.732 (equipo B). Por tanto el equipo A cumplió en un nivel de bajo a medio, mientras que el equipo $\mathrm{B}$ cumplió medianamente.

Tabla 4: Grado de cumplimiento del problema planteado.

\begin{tabular}{|l|c|c|}
\hline \multirow{2}{*}{ Requisitos del Problema } & Grado de Cumplimiento \\
\cline { 2 - 4 } & \multicolumn{2}{|c|}{ Equipo } \\
\hline $\begin{array}{l}\text { Balancear cada una de las ecuaciones químicas por el método algebraico desarrollando } \\
\text { un programa en MATLAB }{ }^{\circledR}\end{array}$ & 3 \\
\hline Comentar acerca de las limitaciones del método algebraico $^{*} 3$ \\
\hline Comparar este método de balanceo con otros métodos químicos & 0 & 0 \\
\hline Promedio & 2 & 3 \\
\hline Desviación estándar & 1.667 & 2.000 \\
\hline
\end{tabular}

Los equipos $\mathrm{A}$ y $\mathrm{B}$ presentaron un breve resumen redactado con un orden claro de ideas respecto al objetivo del trabajo y los resultados obtenidos. Ambos equipos no incluyeron un marco teórico sobre los conceptos en los que se basaron para resolver el problema planteado. La metodología de los equipos fue clara, pero sin un lenguaje técnico adecuado. Respecto a sus conclusiones, ambos equipos argumentaron mejor los resultados con ayuda del programa MATLAB ${ }^{\circledR}$, mientras que omitieron conclusiones acerca del balanceo de ecuaciones químicas por los métodos químicos. En su reporte presentan las referencias bibliográficas a las que recurrieron, sin embargo, estas no fueron referenciadas correctamente, esto debido en gran parte a que no incluyeron un marco teórico. En la Tabla 5 se presenta el grado de cumplimiento del formato del reporte solicitado a los alumnos sobre el problema planteado. Los equipos A y B tienen un nivel de cumplimiento de bajo a medio mostrando un promedio de 1.667 y 1.833 respectivamente, con una desviación estándar de 1.032 y 1.169 respectivamente.

Tabla 5: Grado de cumplimiento del formato del reporte entregado.

\begin{tabular}{|l|c|c|}
\hline \multirow{2}{*}{ Sección } & \multicolumn{2}{|c|}{ Equipo } \\
\hline Resumen & 2 & A \\
\hline Marco teórico & 0 & 2 \\
\hline Metodología & 2 & 3 \\
\hline Resolución del ejercicio & 3 & 3 \\
\hline Conclusiones & 2 & 2 \\
\hline Referencias bibliográficas & 1 & 2 \\
\hline Promedio & 1.667 & 1.833 \\
\hline Desviación estándar & 1.032 & 1.169 \\
\hline
\end{tabular}


El promedio del grado de desarrollo de las capacidades de los equipos A y B es 1.627 y 1.750 con una desviación estándar de 0.757 y 0.957 respectivamente. Por lo anterior, podemos comentar que el grado de desarrollo de las capacidades del mundo laboral de ambos equipos se encuentra entre bajo y medio. Lo cual corrobora lo reportado por Ramírez et al. (2013) y Felder et al. (2000), quienes mencionan de forma indirecta que el desarrollo de habilidades y capacidades no solo dependen del instructor sino también de la actitud de los estudiantes para desarrollar las capacidades demandadas por el mundo laboral. Sin embargo, implementando técnicas de aprendizaje activo en sus versiones de aprendizaje colaborativo, aprendizaje cooperativo, basado en problemas y proyectos es una buena forma de impulsar y fomentar dichas capacidades laborales.

Tabla 6: Grado de desarrollo de las capacidades.

\begin{tabular}{|l|c|c|}
\hline \multirow{2}{*}{ Capacidad } & \multicolumn{2}{|c|}{ Equipo } \\
\cline { 2 - 3 } & $A$ & $B$ \\
\hline Aprendizaje & 2.5 & 3 \\
\hline Personales & 1 & 1 \\
\hline Autonomía & 1 & 1 \\
\hline Valores & 2 & 2 \\
\hline Promedio & 1.627 & 1.750 \\
\hline Desviación estándar & 0.757 & 0.957 \\
\hline
\end{tabular}

\section{COMENTARIOS DE LOS ESTUDIANTES}

Dentro de los comentarios positivos de los estudiantes se incluyen que el aprendizaje activo fomenta el trabajo entre compañeros, del cual aprenden mucho, porque los compañeros aportan varios puntos de vista. Así mismo, fomenta la educación interdisciplinaria, ya que aplican conceptos de distintas áreas en una misma asignatura. Además, los hace trabajar por sí mismos, de tal manera que se sienten útiles al aportar puntos de vista personales. En cuanto a comentarios negativos un alumno declara desde su punto de vista no estuvo de acuerdo con el trabajo en equipo dado que no todos sus compañeros contribuyeron en el proceso de enseñanza-aprendizaje; además menciono que prefiere el método de enseñanza tradicional. Otro alumno manifestó sentirse presionado dado que no sabe administrar sus tiempos de estudio.

\section{EJEMPLO DEL MÉTODO REDOX}

En esta sección presentamos un ejemplo del método REDOX con una serie de pasos a seguir. En la figura 1 se muestra un ejemplo de diagrama de flujo para el desarrollo del código en MATLAB $^{\circledR}$ que proporcione la solución a los sistemas de ecuaciones lineales que dan solución al balanceo de la ecuación química.

Con el fin de ejemplificar y comparar los métodos de balanceo de reacciones químicas (método REDOX y método algebraico) tomamos como base la reacción química 1 de la Tabla 2. Comencemos con el balanceo por el método REDOX. En la Tabla 7 se presentan los pasos iniciales del balanceo de la ecuación 1, es decir, la determinación de los estados de oxidación de cada una de las especies químicas que intervienen en la reacción (paso 1) y el establecimiento de las semi-reacciones REDOX (paso 2); como se observa en dicha tabla los estados de oxidación del $\mathrm{N}$ (elipse con línea azul punteada) y del Mn (elipse con línea roja con guiones) son los únicos que cambian, por lo tanto tenemos identificadas las semi-reacciones de oxidación y reducción.

Tabla 7: Matriz de estados de oxidación de la ecuación química 1.

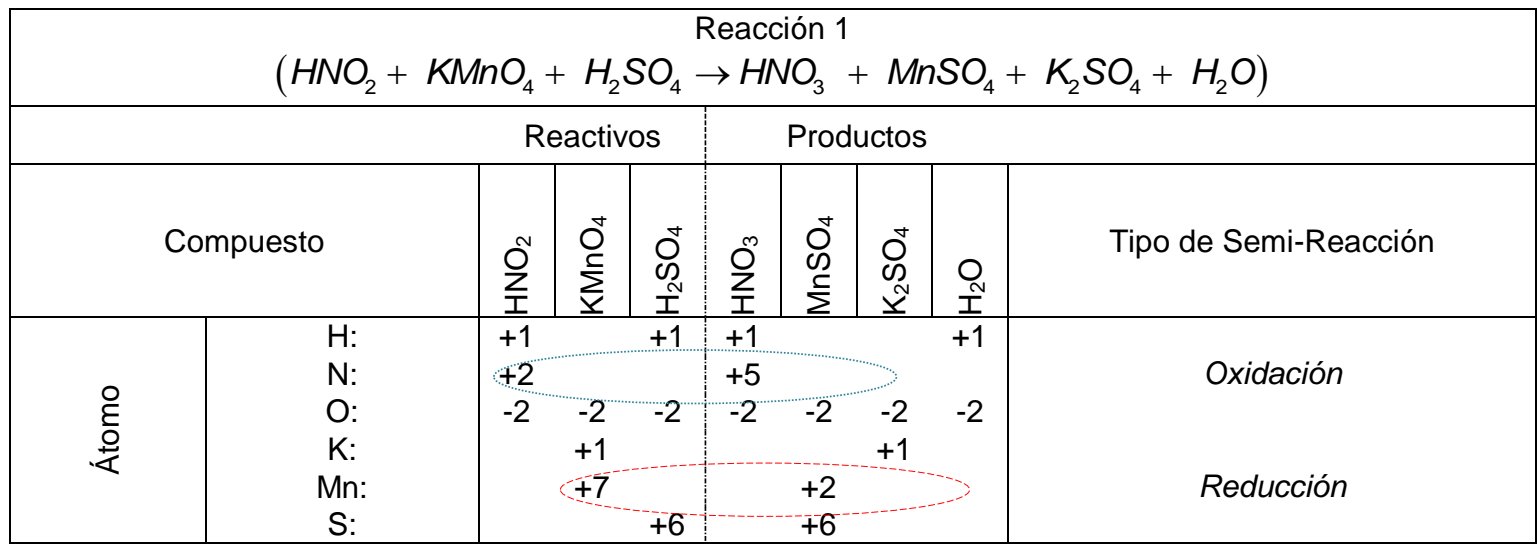




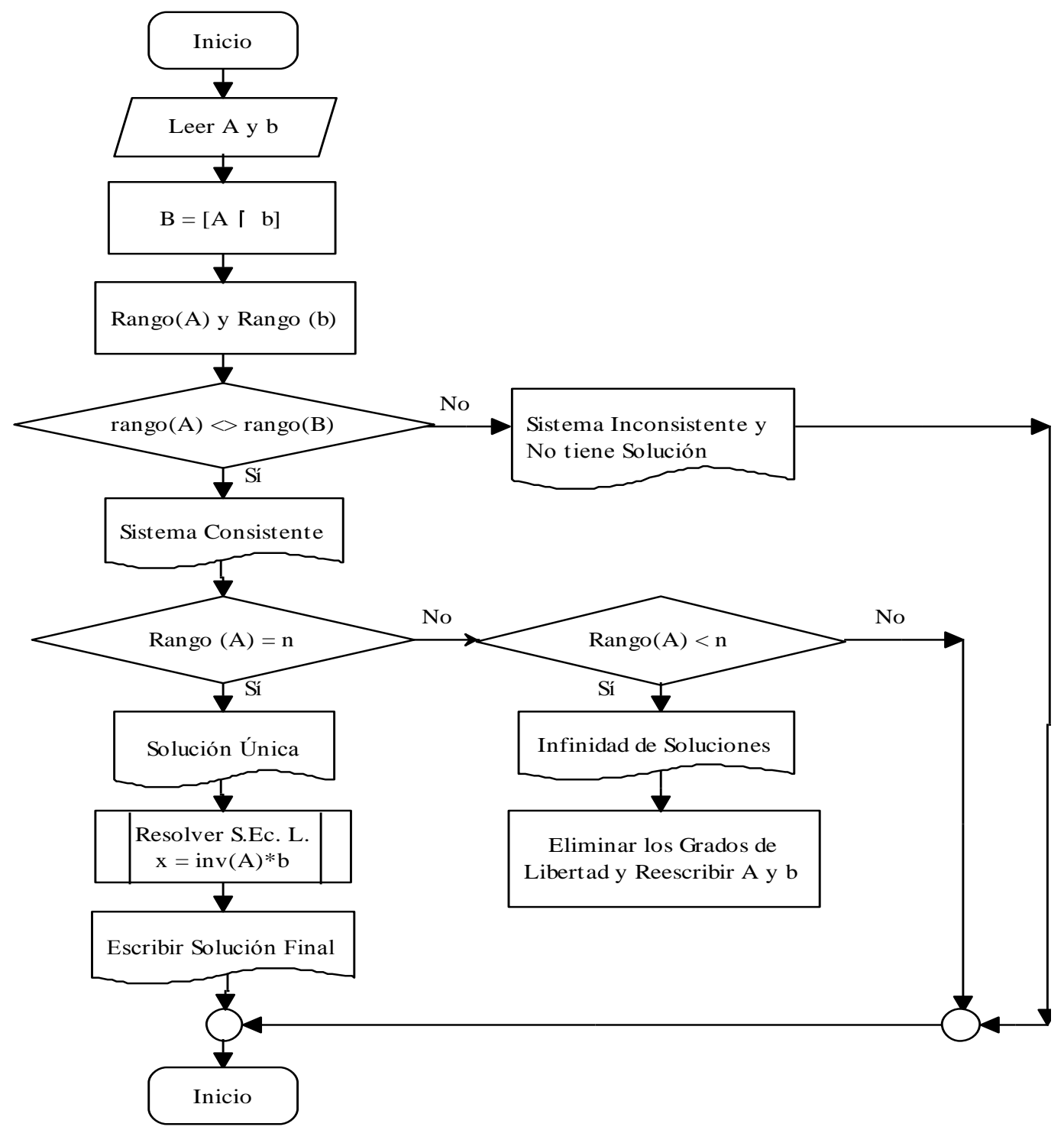

Fig. 1: Diagrama de flujo para la solución de sistemas de ecuaciones lineales.

En la Tabla 8 se muestran en forma sintética los pasos fundamentales del balaceo de la reacción química 1 por el método REDOX.

Tabla 8: Pasos del método REDOX para el balanceo de la ecuación química 1.

\begin{tabular}{|c|c|}
\hline Pasos & Acción \\
\hline \multirow{2}{*}{3} & $\mathrm{NO}_{2}^{-} \rightarrow \mathrm{NO}_{3}^{-} \quad$ (agente reductor $)$ \\
\hline & $\mathrm{MnO}_{4}^{-} \rightarrow \mathrm{Mn}^{+2} \quad$ (agente oxidante $)$ \\
\hline \multirow{2}{*}{4} & $\mathrm{NO}_{2}^{-}+\mathrm{H}_{2} \mathrm{O} \rightarrow \mathrm{NO}_{3}^{-}+2 \mathrm{H}^{+}$ \\
\hline & $\mathrm{MnO}_{4}^{-}+8 \mathrm{H}^{+} \rightarrow \mathrm{Mn}^{+2}+4 \mathrm{H}_{2} \mathrm{O}$ \\
\hline \multirow{2}{*}{5} & $\mathrm{NO}_{2}^{-}+\mathrm{H}_{2} \mathrm{O} \rightarrow \mathrm{NO}_{3}^{-}+2 \mathrm{H}^{+}+2 \mathrm{e}^{-}$ \\
\hline & $\mathrm{MnO}_{4}^{-}+8 \mathrm{H}^{+}+5 e^{-} \rightarrow \mathrm{Mn}^{+2}+4 \mathrm{H}_{2} \mathrm{O}$ \\
\hline \multirow{3}{*}{6 y 7} & $5 \mathrm{NO}_{2}^{-}+5 \mathrm{H}_{2} \mathrm{O} \rightarrow 5 \mathrm{NO}_{3}^{-}+\underline{10 \mathrm{H}^{+}}+10 \mathrm{e}^{-}$ \\
\hline & $2 \mathrm{MnO}_{4}^{-}+\underline{16 \mathrm{H}^{+}}+10 \mathrm{e}^{-} \rightarrow 2 \mathrm{Mn}^{+2}+8 \mathrm{H}_{2} \mathrm{O}$ \\
\hline & $5 \mathrm{HNO}_{2}+2 \mathrm{MnO}_{4}^{-}+6 \mathrm{H}^{+} \rightarrow 5 \mathrm{HNO}_{3}+2 \mathrm{Mn}^{+2}+3 \mathrm{H}_{2} \mathrm{O}$ \\
\hline 8 & $5 \mathrm{HNO}_{2}+2 \mathrm{KMnO}_{4}+3 \mathrm{H}_{2} \mathrm{SO}_{4} \rightarrow 5 \mathrm{HNO}_{3}+2 \mathrm{MnSO}_{4}+\mathrm{K}_{2} \mathrm{SO}_{4}+3 \mathrm{H}_{2} \mathrm{O}$ \\
\hline
\end{tabular}


En la Tabla 9 se muestra la matriz atómica de la ecuación química 1. Así mismo, se identifica la matriz $A$ y el vector $b$ para posteriormente ordenarlo en forma matricial (estado de espacio).

Tabla 9: Matriz atómica de la ecuación química 1.

\begin{tabular}{|c|c|c|c|c|c|c|c|c|c|c|}
\hline \multicolumn{11}{|c|}{ Reacción $1\left(\mathrm{HNO}_{2}+\mathrm{KMnO}_{4}+\mathrm{H}_{2} \mathrm{SO}_{4} \rightarrow \mathrm{HNO}+\mathrm{MnSO}_{4}+\mathrm{K}_{2} \mathrm{SO}_{4}+\mathrm{H}_{2} \mathrm{O}\right)$} \\
\hline \multicolumn{2}{|c|}{ Compuesto } & $\mathrm{HNO}_{2}$ & $\mathrm{KMnO}_{4}$ & $\mathrm{H}_{2} \mathrm{SO}_{4}$ & $\mathrm{HNO}_{3}$ & $\mathrm{MnSO}_{4}$ & $\mathrm{~K}_{2} \mathrm{SO}_{4}$ & $\mathrm{H}_{2} \mathrm{O}$ & $\underset{\sim}{x}$ & Valor Asignado \\
\hline \multirow{6}{*}{ 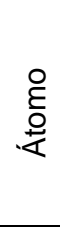 } & $\mathrm{H}:$ & 1 & 0 & 2 & -1 & 0 & 0 & -2 & $x_{1}$ & \\
\hline & $\mathrm{N}:$ & 1 & 0 & 0 & -1 & 0 & 0 & 0 & $x_{2}$ & \\
\hline & O: & 2 & 4 & 4 & -3 & -4 & -4 & -1 & $x_{3}$ & \\
\hline & $\mathrm{K}:$ & 0 & 1 & 0 & 0 & 0 & -2 & 0 & $x_{4}$ & \\
\hline & $\mathrm{Mn}:$ & 0 & 1 & 0 & 0 & -1 & 0 & 0 & $x_{5}$ & \\
\hline & S: & 0 & 0 & 1 & 0 & -1 & -1 & 0 & $x_{6}$ & \\
\hline \multicolumn{3}{|c|}{$\underset{\sim}{A x}=\underset{\sim}{b}$} & & & 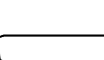 & & & $-b$ & $x_{7}$ & $\leftarrow 3$ \\
\hline
\end{tabular}

En la Figura 2 se muestra la ventana de Command Window en MATLAB ${ }^{\circledR}$, la cual contiene resultados arrojados del programa diseñado para el balanceo de ecuaciones químicas. Esta ventana contiene el número de incógnitas, el rango $(A)$, el rango $(B=A \mid b)$, la caracterización del sistema y la descripción detallada del tipo de solución.

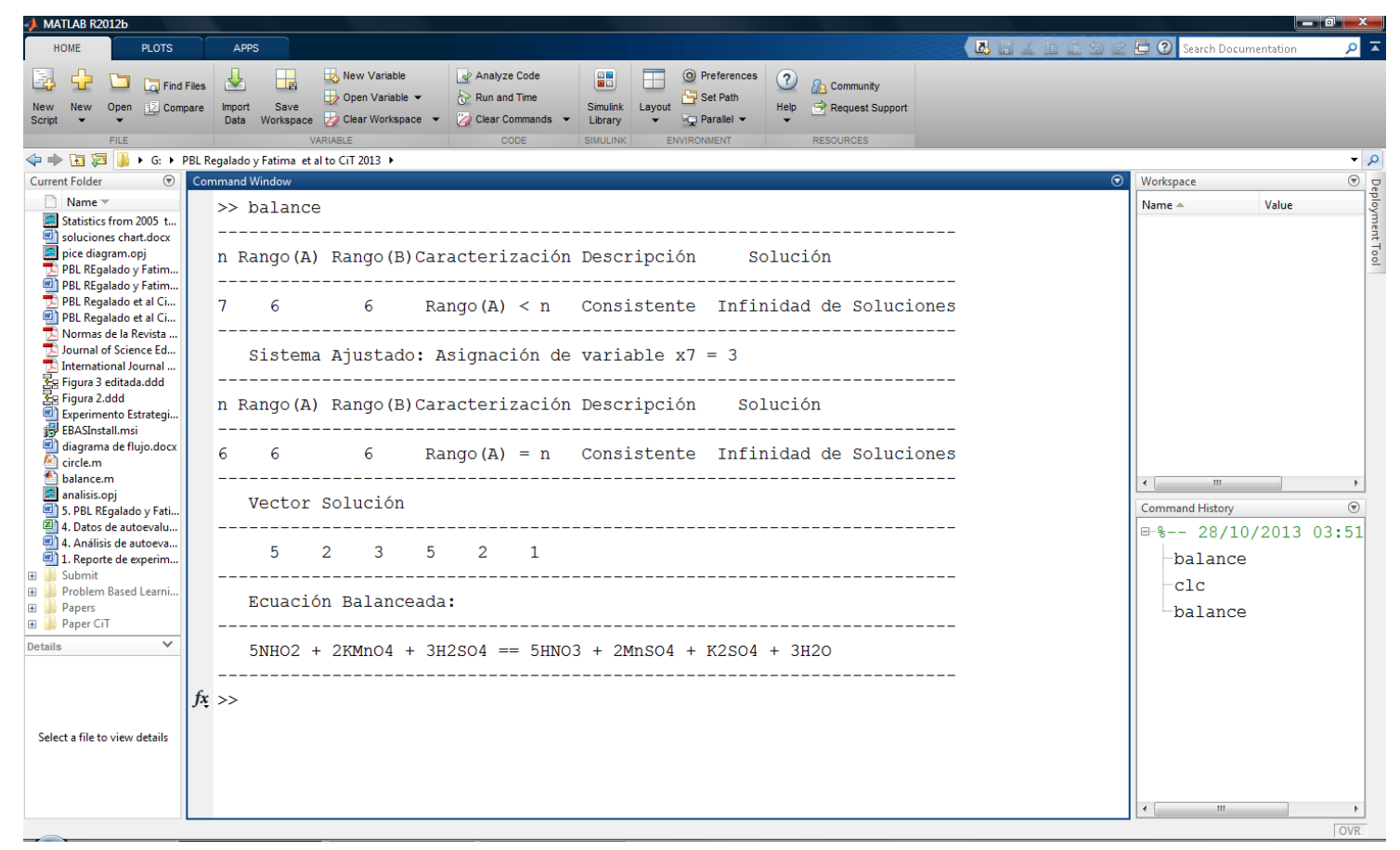

Fig. 2: Ventana de Command Window de MATLAB ${ }^{\circledR}$ de la solución de la ecuación química 1.

Como alternativa a lo anterior, con fines didácticos y rápida forma de balancear ecuaciones químicas, el facilitador puede descargar en internet un trial de 30 días del software titulado EBAS-equation balancer ${ }^{\circledR}$ (disponible en: http://www.chembuddy.com/?left=EBAS\&right=balancing-chemical-equations).

En la Figura 3 se muestra la solución de la ecuación química 1 con el fin de ejemplificar. En dicha Figura, (i) los óvalos morados muestran los iconos para poder adicionar reactivos y productos; (ii) el óvalo rojo indican donde se deben introducir los compuestos químicos; estos pueden ser ingresados empleando la base de datos para seleccionar los reactivos y los productos o bien ingresar las formulas químicas por el teclado; (iii) el ovalo verde muestra la ecuación química balanceada. Nótese que el software calcula los pesos moleculares inmediatamente y que puede realizar cálculos estequiométricos, entre otros cálculos. 


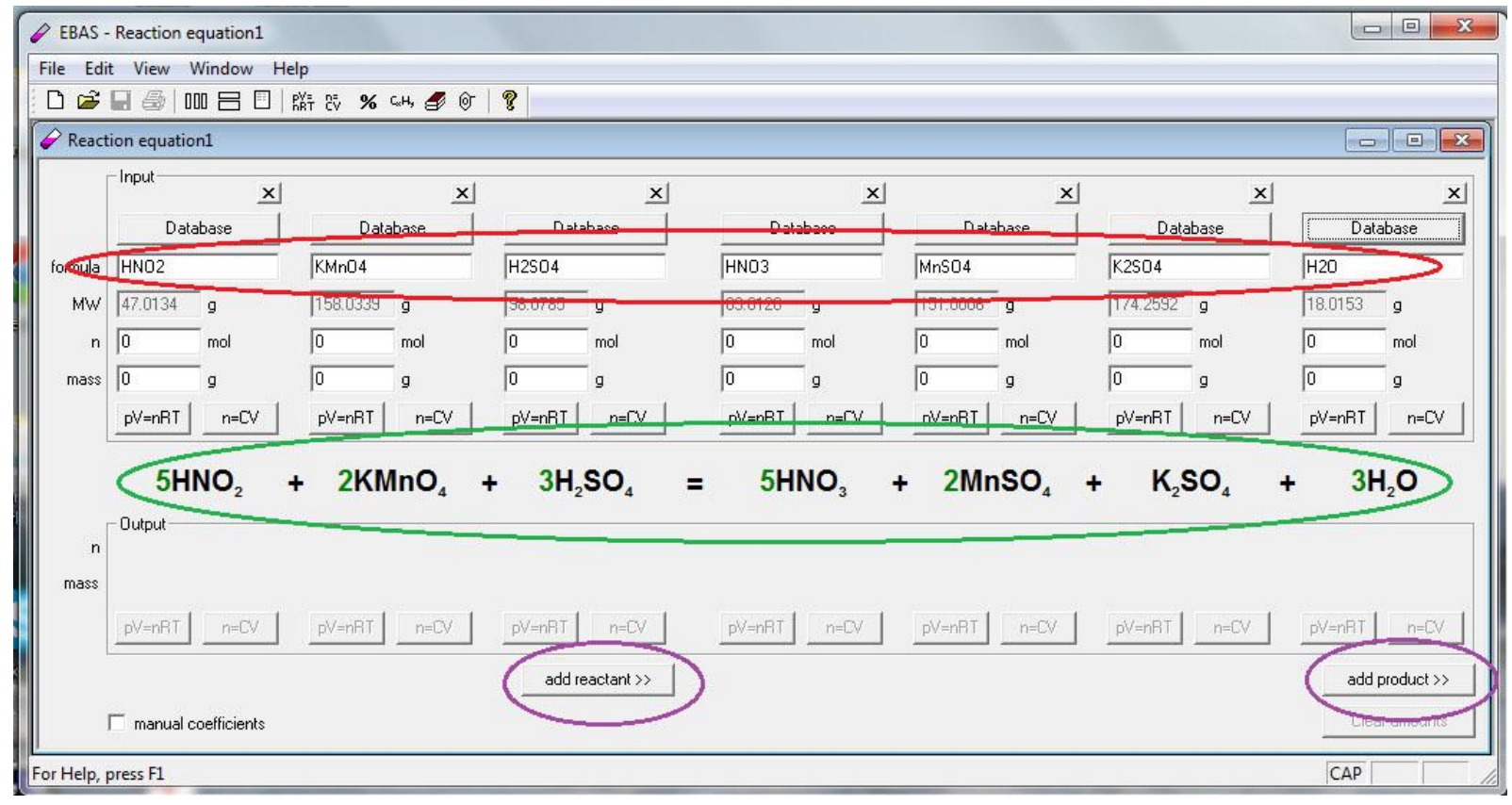

Fig. 3: Ejemplo del software EBAS-equation balancer ${ }^{\circledR}$.

Adicionalmente a lo anterior Risteski (2008) presenta un nuevo método generalizado de matriz inversa para el balanceo de ecuaciones químicas. El método se basa en la solución de una matriz homogénea de ecuaciones usando la matriz pseudoinversa de von Neumann. El método funciona apropiadamente y no tiene limitaciones. Debido a que incluye el balanceo de ecuaciones químicas que poseen números de oxidación fraccionarios.

\section{CONCLUSIONES}

De los argumentos presentados, de la discusión y análisis de resultados y de los antecedentes de la literatura expuestos a través del artículo, se obtienen las siguientes conclusiones:

La actividad de aprendizaje activo propuesta en este trabajo fue medianamente exitosa ya que el grado de integración promedio de los equipos es del 2.75 , el cual se considera de medio a alto. Sin embargo, el promedio del grado de desarrollo de las capacidades del mundo laboral de los equipos es del 1.688, el cual se considera de bajo a medio.

La actividad de aprendizaje activo apoyó al incremento en el número de alumnos acreditados para el ciclo escolar 2013 en los cursos de química y computación, ya que el porcentaje de alumnos aprobados fue del $83.33 \%$ y $100 \%$ respectivamente.

\section{REFERENCIAS}

Arnaldos, J., Planas, E., Darbra, R.M., Casal J., Pastor, E., y Vílchez, J.V., Risk analysis active learning through the investigation of real cases, Process Safety and Environmental Protection, 9(5), 430-435 (2012).

Açikgöz Ü, K. Aktifögrenme, Izmir: Bilis (2006).

Blasco-Arcas, L., Buil, I., Hernández-Ortega, B., y Sese, F.J., Using clickers in class. The role of interactivity, active collaborative learning and engagement in learning performance, Computers \& Education, 62(1), 102110 (2013).

Blottnitz, H.V., Promoting active learning in sustainable development: experiences from a 4th year chemical engineering course, Journal of Cleaner Production, 14(9-11), 916-923 (2006).

Bullard, L.G., y Felder, y R.M., A Student-Centered Approach to Teaching Material and Energy Balances, 2. Course Delivery and Assessment, Chem. Engr. Education, 41(3), 167-176 (2007). 
Declaración de Bolonia Junio de 1999, Espacio Europeo de Enseñanza Superior. http://eees.umh.es/contenidos/Documentos/DeclaracionBolonia.pdf. Acceso: 20 de Noviembre (2013).

El-Naas M. H., Teaching water desalination through active learning, Education for Chemical Engineers, 6(4), e97-e102 (2011).

Evans, G.M., Galvin, K.P., y Doroodchi, E., Introducing quantitative life cycle analysis into the chemical engineering curriculum, Education for Chemical Engineers, 3(1), e57-e65 (2008).

Felder, R.; Woods, D.; Stice J.; Rugarcía, A., The Future of Engineering Education II. Teaching methods that work, Chem. Eng. Education, 34 (1), 26-39 (2000).

Felder, R.M., y Brent R., Learning by doing, Chemical Engineering Education, 37(4), 282-283 (2003).

Felder, R.M., y Brent R., Active Learning: an Introduction, ASQ Higher Education Brief, 2(4), 1-5 (2009).

Gavilán I., S. Cano., y Aburto S., Diseño de herramientas didácticas basado en competencias para la enseñanza de la química ambiental, Educ. quím., 24(3), 298-308 (2013).

González, V.M., y González, R.M, Competencias genéricas y formación profesional: un análisis desde la docencia universitaria, Revista Iberoamericana de Educación, 47(3), 185-209 (2008).

González, A.M., Serrano, L., Llano-Ponte, R., y Labidi J., Evaluation of the biomass fractionation capability of the ultrafiltration permeate: A learning project for chemical engineering students, Education for Chemical Engineers, 7(4), e241-e246 (2012).

Keyser, M.W., Active learning and cooperative learning: understanding the difference and using both styles effectively, Research Strategies, 17(1), 35-44 (2000).

Middleton, R., Active learning and leadership in an undergraduate curriculum: How effective is it for student learning and transition to practice?, Nurse Education in Practice, 13(2), 83-88 (2013).

Prince M., Does Active Learning Work? A Review of the Research, J. Engr. Education, 93(3), 223-231 (2004).

Ramaswamy, S., Harris, I., y Tschirner, U., Student peer teaching: an innovative approach to instruction in science and engineering education, J. Sci. Educ. Technol., 10(2), 165-171 (2001).

Ramírez, J.A., Alejo, M.G., Jiménez, R., y Marmolejo S., Percepción de los Estudiantes de Ciencias Químicas sobre sus Equipos de Trabajo, Formación Universitaria, 6(3), 3-12 (2013).

Risteski, I.C., A New Generalized Matrix Inverse Method for Balancing Chemical Equations and their Stability, Bol. Soc. Quím. Méx., 2(3), 104-115 (2008).

Tedesco-Schneck, M., Active learning as a path to critical thinking: Are competencies a roadblock?, Nurse Education in Practice, 13(1), 58-60 (2013). 
